\title{
Carvacrol protects against spinal cord injury in rats via suppressing oxidative stress and the endothelial nitric oxide synthase pathway
}

\author{
ZHEN-SONG JIANG ${ }^{1 *}$, ZHI-CHEN PU ${ }^{2 *}$ and ZHEN-HAI HAO ${ }^{1}$ \\ ${ }^{1}$ Department of Orthopedics, Provincial Hospital Affiliated to Shandong University, Jinan, Shandong 250014; \\ ${ }^{2}$ Department of Clinical Pharmacy, Wannan Medical College, Yijishan Hospital, Wuhu, Anhui 241001, P.R. China
}

Received April 7, 2014; Accepted January 26, 2015

DOI: $10.3892 / \mathrm{mmr} .2015 .4045$

\begin{abstract}
Carvacrol (CAR) is a naturally occurring phenolic monoterpene and has been demonstrated to possess a spectrum of pharmacological actions. The present study was designed to assess the neuroprotection of CAR against spinal cord injury (SCI) in rats and to identify the underlying mechanisms. SCI was induced using the modified weight-drop method in Wistar rats. CAR or saline was administered at doses of 25, 50 and $100 \mathrm{mg} / \mathrm{kg}$ for 46 days. Neuronal function following SCI was evaluated using the Basso, Beattie and Bresnahan (BBB) locomotor rating scale. Spinal cord edema was assessed by measuring the water content in spinal cord tissues. The oxidative indicators, including malondialdehyde, catalase, superoxide dismutase glutathione peroxidase and 8 -isoprotane as well as endothelial nitric oxide synthase (eNOS) activity and caspase-3 were measured using corresponding commercial kits. The protein expression of eNOS and $\mathrm{B}$ cell lymphoma-2 (Bcl-2) as well as Bcl-2-associated $\mathrm{X}$ protein (Bax) was analyzed by western blot analysis. The SCI-induced rats demonstrated marked reductions in BBB scores. CAR treatment recovered neurological function with decreasing $\mathrm{BBB}$ scores. CAR was found to have inhibitory effects on the water content in the spinal cord, oxidative stress, eNOS, nitric oxide production and apoptosis-associated molecules, including Bax and caspase-3 as well as promoting Bcl-2 expression in SCI-induced rats. These results suggested that CAR protects against SCI via mediating oxidative stress and the eNOS signaling pathway.
\end{abstract}

Correspondence to: Professor Zhen-Hai Hao, Department of Orthopedics, Provincial Hospital Affiliated to Shandong University, 9677 Jingshi Road, Jinan, Shandong 250014, P.R. China

E-mail: zhezhudr@163.com

*Contributed equally

Key words: carvacrol, spinal cord injury, oxidative stress, endothelial nitric oxide synthase pathway

\section{Introduction}

It is well established that spinal cord injury (SCI) produces primary damage and triggers a prolonged period of secondary lesion. Among the factors associated with the secondary injury, reactive oxygen species have attracted significant attention for their role in the pathogenesis of SCI (1). Treatment with lecithinized superoxide dismutase, an important antioxidant enzyme, was found to markedly recover SCI-induced motor dysfunction and ameliorate neuronal apoptosis in rats (2). In addition, previous studies have demonstrated that certain traditional Chinese medicinal herbs were able to attenuate neuronal impairment via suppressing oxidative stress in experimental SCI in rats $(3,4)$. These findings imply that oxidative stress may serve as a potential therapeutic target for the amelioration of SCI.

Nitric oxide (NO) is an important endothelium-derived relaxing factor involved in the pathophysiology of SCI. It is well established that NO is produced from the guanidine group of L-arginine by three types of nitric oxide synthase (NOS) enzymes, including endothelial NOS (eNOS), neuronal NOS and inducible NOS (5). Among these isoforms, eNOS is the most important sub-group and increased eNOS activity has been demonstrated to generate significant quantities of NO, subsequently exacerbating the damage following SCI (5).

Carvacrol (CAR) is a natural monoterpenoid phenol compound extracted from the essential oil of the family Lamiaceae, which includes the genera Origanum and Thymus (6). Substantial evidence has demonstrated that CAR possesses diverse biological activities, including antioxidative (7) and anti-apoptotic (8) properties. Two previous studies illustrated that CAR was able to alleviate oxidative damage in rat models of acute myocardial infarction (8) and streptozotocin-induced diabetes (9). However, to the best of our knowledge, there are no studies on the effects of CAR against SCI in rats to date. Furthermore, as oxidative stress and the eNOS signaling pathway are important in the amelioration of SCI, it was hypothesized that they are involved in the neuroprotective effects of CAR. The present study aimed to assess the neuroprotective potential of CAR in SCI-induced rats and examine whether this neuroprotection involves oxidative stress and eNOS pathways. 


\section{Materials and methods}

Animals. Wistar rats weighing $220-260 \mathrm{~g}$ were obtained from the Animal Centre of Beijing (Beijing, China). They were kept in a standard environment and allowed free access to water and food. Experimental protocols were performed in accordance with the guidelines of the Care and Use of Laboratory Animals of the Provincial Hospital Affiliated to Shandong University (Jinan, China). The study was approved by the ethics committee of the Provincial Hospital Affiliated to Shandong University (Jinan, China).

Drugs and reagents. CAR (with a purity $>98 \%$ ) was purchased from Sigma-Aldrich (St. Louis, MO, USA). Commercial kits for malondialdehyde (MDA), catalase (CAT), superoxide dismutase (SOD), glutathione peroxidase (GSH-Px) and eNOS were obtained from Nanjing Jiancheng Biotechnology Institute (Nanjing, China). 8-Isoprostane EIA kit (no. 516351) was obtained from Cayman Chemical (Ann Arbor, MI, USA). Other reagents were all of analytical grade.

Establishment of an SCI rat model and drug administration. The rat model of SCI was prepared as described previously with minor modifications (10). Briefly, the spinal cord was injured at the thoracic level 10 (T10) following an established spinal cord compression model. The skin of rats above the vertebral column was incised and a laminectomy at vertebral level T10 was performed. The dorsal cord surface was exposed with the dura remaining intact. Rats were assigned to five groups: i) Sham group (Sham; $n=10$ ), which experienced sham surgery but no trauma (physiological saline $0.1 \mathrm{ml} / 100 \mathrm{~g}$, i.p.); ii) SCI group $(\mathrm{n}=10)$, which underwent spinal cord injuries and received saline (physiological saline $0.1 \mathrm{ml} / 100$ g, i.p.); iii) CAR (25) group; iv) CAR (50) group and v) CAR (100) group $(n=10)$, which all had spinal cord injuries and were treated with CAR at doses of 25,50 and $100 \mathrm{mg} / \mathrm{kg}$ once a day for 46 consecutive days, respectively. The dosage and dosing frequency of CAR were referred to in a previous study (9).

Evaluation of neuronal function recovery. The motor recovery in SCI rats was evaluated by a locomotor rating scale between 0 (complete paralysis) and 21 (normal locomotion) developed by Basso, Beattie and Bresnahan (BBB) (11).

Assessment of water content in spinal cord tissues. Spinal cord edema was assessed by measuring the water content in spinal cord tissues. Following treatment with CAR for 46 days, the impaired spinal cords were dried at $80^{\circ} \mathrm{C}$ for $48 \mathrm{~h}$ in order to determine the dry weight. Water content of the spinal cords was calculated using the following formula: Spinal cord water content $(\%)=$ (wet weight - dry weight) / wet weight $\mathrm{x} 100 \%$.

Measurement of MDA level and the activity of CAT, SOD and GSH-Px. The oxidative markers, including MDA level and the activities of CAT, SOD and GSH-Px in spinal cord tissues were detected using corresponding commercial kits (Nanjing Jiancheng Bioengineering Institute). Detection of plasma 8-isoprostane levels was performed using the 8-Isoprostane EIA kit.



Figure 1. Chemical structure of carvacrol.

Immunoblotting. Following treatment with CAR for 46 consecutive days, the injured spinal cord tissues were homogenized in ice-cold lysis buffer (50 mM Tris- $\mathrm{HCl}, 150 \mathrm{mM} \mathrm{NaCl}, 10 \%$ glycerol, $1 \%$ Nonidet P-40, 5 mM EDTA and $1 \mathrm{mM}$ phenylmethylsulfonyl fluoride). Supernatant was collected following centrifugation at $12,000 \mathrm{xg}$ for $20 \mathrm{~min}$ and protein quantification was conducted using a BCA kit (Beyotime Institute of Biotechnology, Shanghai, China). Protein (60 $\mu \mathrm{g})$ was separated by electrophoresis on 8 or $10 \%$ SDS-polyacrylamide gels and transferred onto nitrocellulose membranes (Millipore, Billerica, MA, USA). The membranes were probed with the following primary antibodies: Rabbit anti-eNOS (SAB1305369; 1:1,000; Sigma-Aldrich), rabbit anti-Bcl-2-associated X protein (Bax; sc-101874; 1:200; Santa Cruz Biotechnology, Inc., Santa Cruz, CA, USA), rabbit anti-B cell lymphoma-2 (Bcl-2; sc-492; 1:200; Santa Cruz Biotechnology, Inc.) and mouse anti-GAPDH (KC-5G4; 1:2,000; Zhejiang Kangchen Biotech Co., Ltd., Hangzhou, China), overnight at $4^{\circ} \mathrm{C}$. Following washing with phosphate-buffered saline, they were then incubated with horseradish peroxidase-conjugated goat anti-rabbit antibody (sc-45101; 1:5,000; Santa Cruz Biotechnology, Inc.) or goat anti-mouse antibody (sc-2075; 1:5,000; Santa Cruz Biotechnology, Inc.) for $2 \mathrm{~h}$. Immunodetection was conducted with an enhanced chemiluminescence kit (Pierce Biotechnology, Inc., Rockford, IL, USA).

Measurement of eNOS activity in spinal cords and plasma NO production. The eNOS activity was measured according to the manufacturer's instructions of the Nitric Oxide Synthase Assay kit (no. S0025; Beyotime Institute of Biotechnology). Additionally, NO production in the plasma was analyzed by measuring the supernatant for nitrite using Griess reagent (Promega Corp., Madison, WI, USA).

Measurement of caspase-3 activity in spinal cord tissues. As a critical molecule in cellular apoptosis, caspase-3 activity was measured by cleavage of chromogenic caspase substrates, Ac-DEVD-pNA. Colorimetric analysis of the quantity of caspase-3 was performed using a spectrophotometer (Alpha-1860S; Shanghai Puyuan Company, Shanghai, China) at a wavelength of $405 \mathrm{~nm}$.

Statistical analysis. All values are presented as the mean \pm standard deviation and were analyzed using SPSS 16.0 software (SPSS, Inc., Chicago, IL, USA). Statistical analysis was performed using one-way analysis of variance (ANOVA) followed by Dunnett's test. $\mathrm{P}<0.05$ was considered to indicate a statistically significant difference. 
Table I. Effects of CAR on the motor function of rats 24,48 and $72 \mathrm{~h}$ after SCI.

\begin{tabular}{lcrrr}
\hline Group & $\mathrm{n}$ & $24 \mathrm{~h}$ & \multicolumn{2}{c}{$48 \mathrm{~h}$} \\
\hline Sham & 10 & $14.21 \pm 1.12$ & $18.11 \pm 1.27$ & $19.88 \pm 1.47$ \\
SCI & 10 & $4.98 \pm 0.87^{\mathrm{a}}$ & $4.18 \pm 0.82^{\mathrm{a}}$ & $4.05 \pm 0.63^{\mathrm{a}}$ \\
CAR $(25 \mathrm{mg} / \mathrm{kg})$ & 10 & $9.78 \pm 0.89^{\mathrm{b}}$ & $10.12 \pm 0.97^{\mathrm{b}}$ & $11.25 \pm 0.76^{\mathrm{b}}$ \\
CAR $(50 \mathrm{mg} / \mathrm{kg})$ & 10 & $11.63 \pm 0.65^{\mathrm{b}}$ & $11.89 \pm 0.75^{\mathrm{b}}$ & $12.04 \pm 0.95^{\mathrm{b}}$ \\
CAR $(100 \mathrm{mg} / \mathrm{kg})$ & 10 & $12.85 \pm 0.96^{\mathrm{b}}$ & $14.15 \pm 0.88^{\mathrm{b}}$ & $16.21 \pm 1.06^{\mathrm{b}}$
\end{tabular}

${ }^{\mathrm{a}} \mathrm{P}<0.01$, versus sham group; ${ }^{\mathrm{b}} \mathrm{P}<0.01$, versus SCI group. CAR, carvacrol; SCI, spinal cord injury.

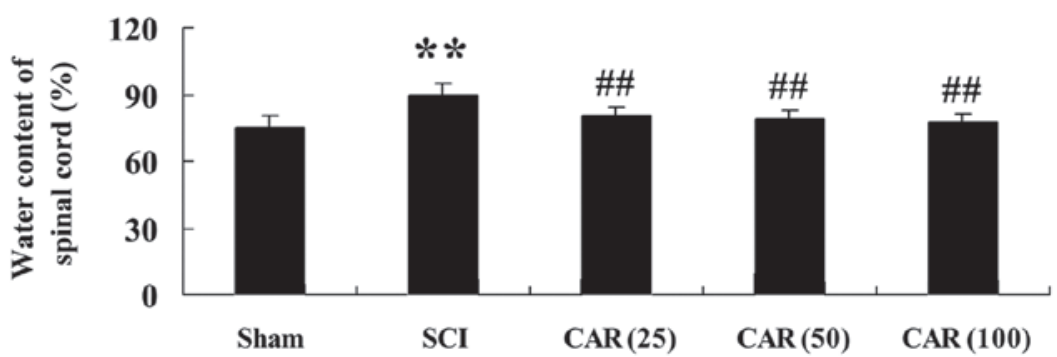

Figure 2. Effects of CAR on the water content of the spinal cord following SCI ( $=10$, mean \pm standard deviation). ${ }^{*} \mathrm{P}<0.01$, compared with the sham group; ${ }^{\# \prime} \mathrm{P}<0.01$, compared with the SCI group. Sham, sham group; SCI, spinal cord injury group; CAR (25), carvacrol (25 mg/kg)-treated group; CAR (50), carvacrol $(50 \mathrm{mg} / \mathrm{kg})$-treated group and CAR (100), carvacrol (100 mg/kg)-treated group. CAR, carvacrol; SCI, spinal cord injury.

\section{Results}

Evaluation of neural function. The chemical structure of CAR is shown in Fig. 1. It was noted that BBB scores in the sham group were $14.21 \pm 1.12,18.11 \pm 1.27$ and $19.88 \pm 1.44$ at 24,48 and $72 \mathrm{~h}$ post-surgery, respectively, as summarized in Table I. By contrast, SCI-induced rats demonstrated severe neurological impairment with marked reductions in BBB scores $(4.98 \pm 0.87$, $4.18 \pm 0.82$ and $4.05 \pm 0.63 ; \mathrm{P}<0.01)$ at the selected time points. However, CAR at doses of 25,50 and $100 \mathrm{mg} / \mathrm{kg}$ significantly improved neurological function $(\mathrm{P}<0.01)$ in injured animals, compared with the SCI model group, particularly at $72 \mathrm{~h}$ post-surgery. Thus, this time point was selected for subsequent investigations.

Assessment of water content in spinal cord tissues of $S C I$-induced rats following CAR treatment. As shown in Fig. 2, there was a marked elevation in water content of the spinal cord $(\mathrm{P}<0.01)$ in the SCI group compared with the sham group. Following treating SCI-induced rats with CAR, the water content in spinal cord tissues was significantly decreased in a dose-dependent manner $(\mathrm{P}<0.01)$ compared with that in the control group.

Effects of CAR on oxidative stress in SCI-induced rats. At $72 \mathrm{~h}$ post-surgery, it was observed that MDA levels were markedly increased and the antioxidant enzymes, including CAT, SOD and GSH-Px were all decreased in spinal cord tissues $(\mathrm{P}<0.01)$, compared with the sham control (Fig. 3A-D). The alterations in MDA content, CAT, SOD and GSH-Px activities were all significantly reversed following administration of CAR (25, 50 and $100 \mathrm{mg} / \mathrm{kg}$ ). In order to confirm the inhibitory effect of
CAR on oxidative damage in SCI-subjected rats, another marker reflecting the oxidative stress, namely, the plasma 8-isoprotane level, was measured in the present study. Fig. 4 shows an evident increase in 8-isoprotane content in the SCI group and administration of CAR dose dependently reversed this phenomenon.

Effects of CAR on the protein expression and activity of eNOS as well as plasma NO concentration following SCI. The present study further examined whether CAR exerted a protective effect through mediating the eNOS pathway. Fig. 5A revealed that western blot analysis with eNOS antibody exhibited an anticipated band of $133 \mathrm{kDa}$. Quantitative analysis demonstrated that there was a significant elevation in the protein level of eNOS $(\mathrm{P}<0.01)$ in injured rats, versus the control group. However, markedly reduced eNOS protein expression was observed following CAR treatment in SCI-induced animals in a dose-dependent manner $(\mathrm{P}<0.01$; Fig. 5B). Similar results were observed in eNOS activity (Fig. 5C). Additionally, NO production was also assessed in the present study. NO production, which was indicated as nitrite formation, was found to be significantly increased following SCI $(\mathrm{P}<0.01)$. CAR treatment markedly reduced the nitrite level $(\mathrm{P}<0.01)$ in a dose-dependent manner, as shown in Fig. 5D.

Effects of CAR on cellular apoptosis following SCI. In order to determine the effect of CAR on cellular apoptosis following $\mathrm{SCI}$, the protein expression of apoptosis-regulated proteins, including Bcl-2 and Bax was detected by western blot analysis. As shown in Fig. 6A, Bcl-2 and Bax exhibited specific bands of 26 and $23 \mathrm{kDa}$, respectively. Following one-way ANOVA analysis, there was evident decreases in $\mathrm{Bcl}-2$ expression and increases in Bax expression following SCI $(\mathrm{P}<0.01)$, versus 
A



C

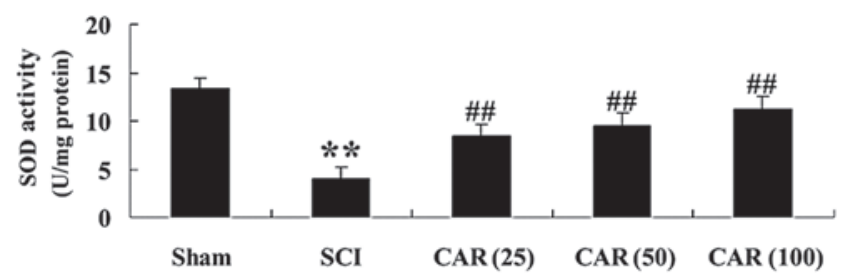

B

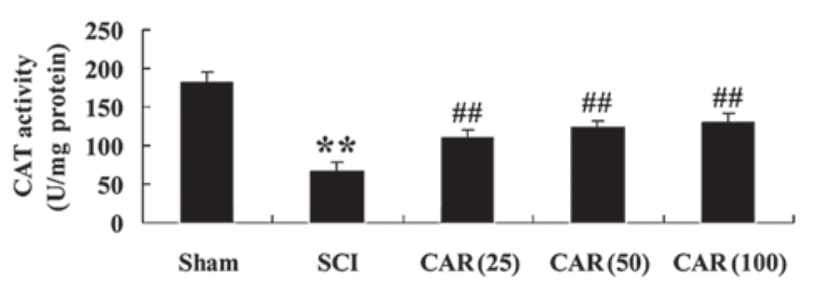

D

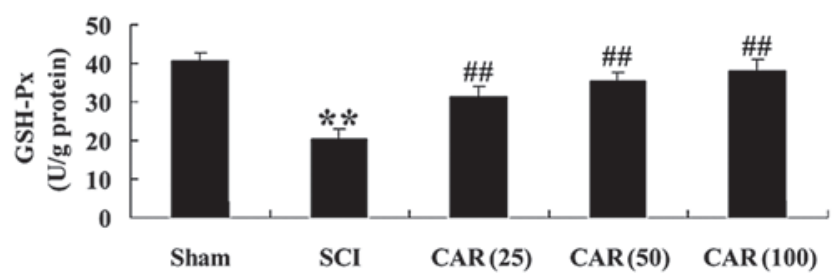

Figure 3. Effects of CAR on the concentration of (A) MDA and on the activities of antioxidant enzymes (B) CAT, (C) SOD and (D) GSH-Px in spinal cord tissues of rats from different groups ( $\mathrm{n}=10$, mean \pm standard deviation). ${ }^{* *} \mathrm{P}<0.01$, compared with the sham group; ${ }^{* *} \mathrm{P}<0.01$, compared with the SCI group. Sham, sham group; SCI, spinal cord injury group; CAR (25), carvacrol (25 mg/kg)-treated group; CAR (50), carvacrol (50 mg/kg)-treated group and CAR (100), carvacrol (100 mg/kg)-treated group. CAR, carvacrol; SCI, spinal cord injury; SOD, superoxide dismutase; GSH-Px, glutathione peroxidase; CAT, catalase; MDA, malondialdehyde.

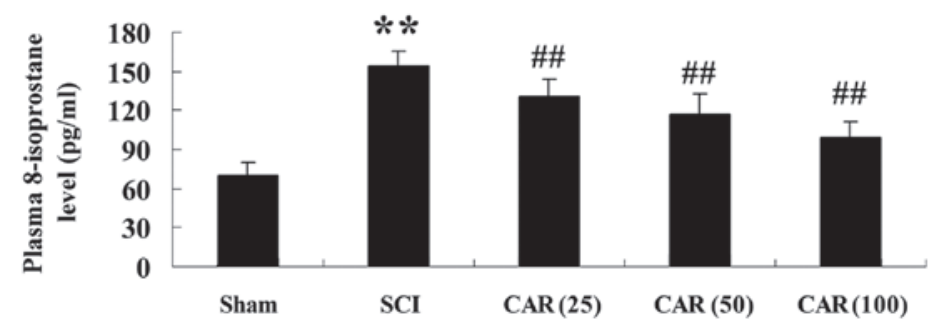

Figure 4. Effects of CAR on the plasma 8-isoprostane level following SCI ( $\mathrm{n}=10$, mean \pm standard deviation). ${ }^{* *} \mathrm{P}<0.01$, compared with the sham group; ${ }^{\# \#} \mathrm{P}<0.01$, compared with the SCI group. Sham, sham group; SCI, spinal cord injury group; CAR (25), carvacrol (25 mg/kg)-treated group; CAR (50), carvacrol $(50 \mathrm{mg} / \mathrm{kg})$-treated group and CAR (100), carvacrol (100 mg/kg)-treated group. CAR, carvacrol; SCI, spinal cord injury.

A
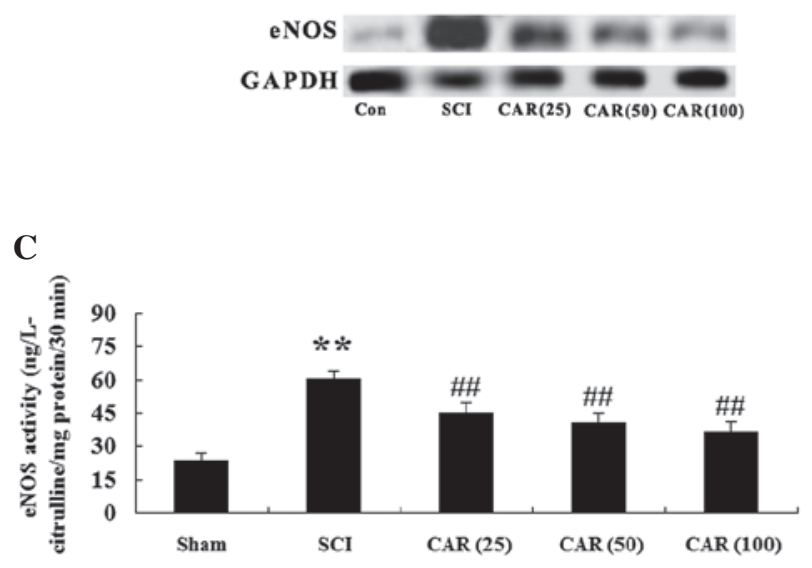

B

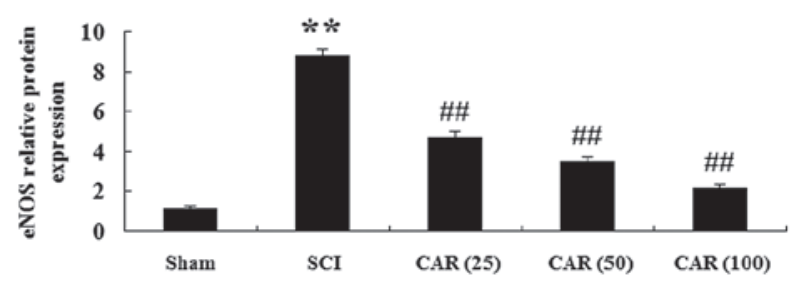

D

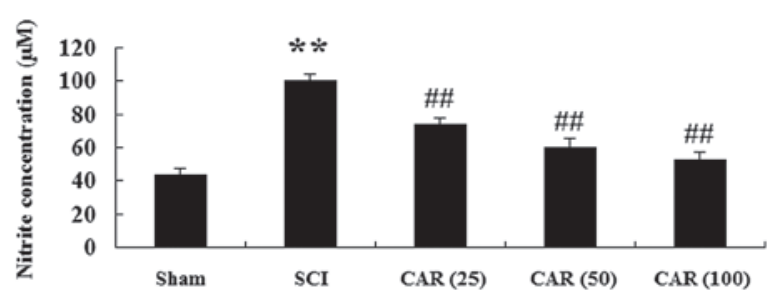

Figure 5. Effects of CAR on the protein level and activity of eNOS as well as plasma NO concentration following SCI ( $\mathrm{n}=10$, mean \pm standard deviation). (A) Representative images of immunoblots with antibodies against eNOS in injured spinal cords from different groups. eNOS: $133 \mathrm{kDa}$; GAPDH: $36 \mathrm{kDa}$. (B) Quantitative analysis of the protein level of eNOS in spinal cords from different groups. The data were normalized to the loading control GAPDH. (C) Measurement of eNOS activity. (D) NO production was detected spectrophotometrically by measuring its metabolite, nitrite. ${ }^{* *} \mathrm{P}<0.01$, compared with the sham group; ${ }^{\# \prime} \mathrm{P}<0.01$, compared with the SCI group. Sham, sham group; SCI, spinal cord injury group; CAR (25), carvacrol (25 mg/kg)-treated group; CAR (50), carvacrol (50 mg/kg)-treated group and CAR (100), carvacrol (100 mg/kg)-treated group. CAR, carvacrol; SCI, spinal cord injury; eNOS, endothelial nitric oxide synthase; NO, nitric oxide. 
A

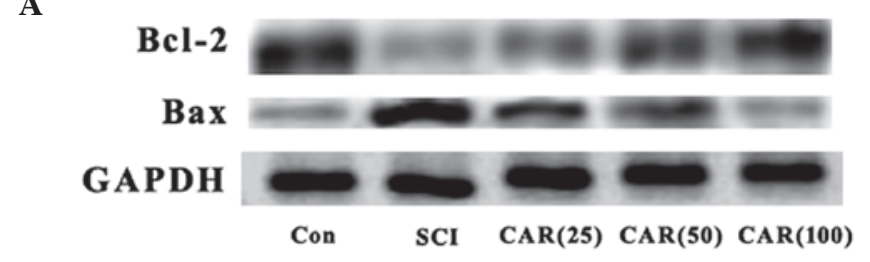

B
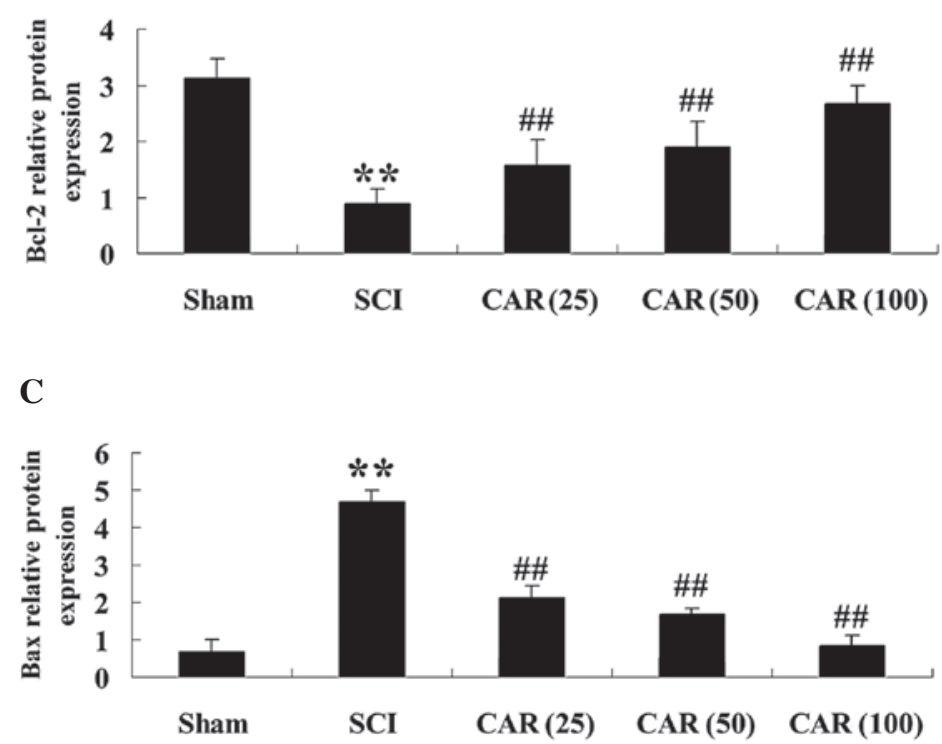

Figure 6. Effects of CAR on the protein expression of Bcl-2 and Bax following SCI (n=10, mean \pm standard deviation). (A) Representative images of immunoblots with antibodies against Bcl-2 and Bax in injured spinal cords from different groups. Bcl-2: $26 \mathrm{kDa}$; Bax: $23 \mathrm{kDa}$; GAPDH: $36 \mathrm{kDa}$. (B and C) Quantitative analysis of the protein levels of Bcl-2 and Bax, respectively in spinal cords from different groups. The data were normalized to the loading control GAPDH. ${ }^{* *} \mathrm{P}<0.01$, compared with the sham group; ${ }^{\# \#} \mathrm{P}<0.01$ compared with the SCI group. Sham, sham group; SCI, spinal cord injury group; CAR (25), carvacrol (25 mg/kg)-treated group; CAR (50), carvacrol (50 mg/kg)-treated group and CAR (100), carvacrol (100 mg/kg)-treated group. SCI, spinal cord injury; CAR, carvacrol; Bcl-2, B cell lymphoma-2; Bax, Bcl-2-associated X protein.

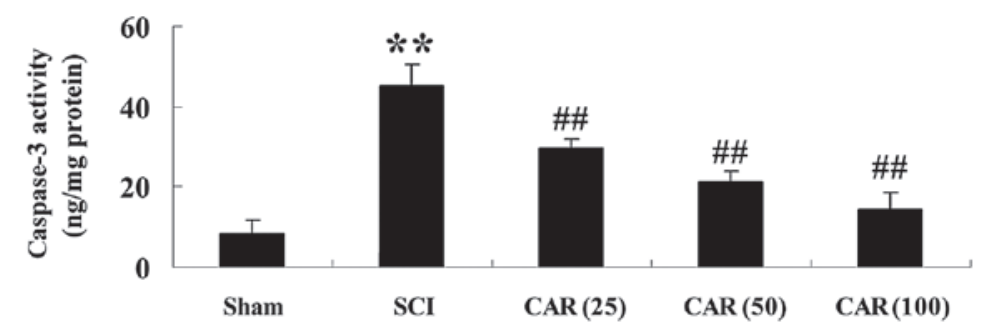

Figure 7. Effects of CAR on caspase- 3 activity following SCI ( $\mathrm{n}=10$, mean \pm standard deviation). ${ }^{* *} \mathrm{P}<0.01$, compared with the sham group; ${ }^{\# \#} \mathrm{P}<0.01$, compared with the SCI group. Sham, sham group; SCI, spinal cord injury group; CAR (25), carvacrol (25 mg/kg)-treated group; CAR (50), carvacrol (50 mg/kg)-treated group and CAR (100), carvacrol (100 mg/kg)-treated group. SCI, spinal cord injury; CAR, carvacrol.

the control. CAR treatment to the SCI-induced rats increased the expression of Bcl-2 and decreased Bax at the protein level in a dose-dependent manner (Fig. 6B and C). The activity of caspase-3, an executive molecule in the apoptotic cascade, was found to be increased in the SCI group $(\mathrm{P}<0.01)$ and CAR significantly suppressed this increase, as shown in Fig. 7.

\section{Discussion}

The major findings of the present study illustrated that CAR facilitated the recovery of motor function in SCI-induced rats and that its neuroprotection may be associated with suppressing oxidative stress and the eNOS signaling pathway.
It has been demonstrated that SCI-induced primary damage is irreversible and appears to not be amenable to neuroprotective therapy. However, the secondary impairment occurs in response to the deleterious substances produced following primary trauma (12). Among these substances, oxidative stress is regarded as an important factor during traumatic SCI. Evidence supported that marked generation of reactive oxygen species was observed in rats following SCI and treatment with antioxidant compounds attenuated edema formation and cellular apoptosis (13). It was previously reported that CAR protected against acute myocardial infarction and diabetes-associated cognitive deficits in rats via antioxidative mechanisms $(8,9)$. In addition, results from the current study demonstrated that CAR markedly decreased 
the concentration of MDA, an important and reliable index for determining the extent of the peroxidation reaction (14), and increased the activities of antioxidant enzymes, including CAT, SOD and GSH-Px as well as reduced the levels of the oxidative marker, 8-isoprotane, following SCI in rats. This implies that the protective role of CAR against SCI may be associated with the inhibition of free radical and oxidant formation.

NO is considered to be one of the major regulators of spinal damage. A previous study revealed that eNOS-derived NO production aggravated the impairment caused by SCI in rats (5). Additionally, a previous study reported the attenuation of injury in rats exposed to traumatic SCI following treatment with rosuvastatin via reducing NO production (15). The present study revealed that the protein expression level and the activity of eNOS together with NO concentration were all elevated in the SCI group. In addition, CAR treatment significantly decreased eNOS levels and correspondingly suppressed the elevated quantity of NO in rats with SCI. CAR was previously found to have NO-scavenging activity (7). Taken together, these findings supported that CAR protects the spinal cord from injury via suppressing eNOS and concomitantly decreasing NO bioavailability.

The secondary lesion caused by SCI can damage spinal neurons and trigger apoptotic cascades. The pharmacological inhibition of apoptosis may function as a potential therapeutic strategy. Targeted retrograde gene delivery of brain-derived neurotrophic factor was reported to inhibit cellular apoptosis and restore neurological function following SCI (16). Bcl-2 family proteins have been demonstrated to be important in the modulation of cellular apoptosis. Under normal circumstances, Bcl-2 itself serves as an anti-apoptotic protein, whereas another member of the family, Bax, acts as a pro-apoptotic molecule (17). The results of the present study demonstrated that the evident reduction of $\mathrm{Bcl}-2$ and increased Bax protein levels were observed in the spinal cord tissues of SCI. However, treatment with CAR dose dependently caused elevated levels of Bcl-2 and reduced Bax protein in SCI-induced rats. In addition, the activity of caspase- 3 , an executioner molecule in the apoptotic signaling pathway, was found to be markedly elevated in rats following SCI and CAR significantly inhibited this index. Collectively, these findings indicated that the neuroprotective action of CAR may involve the regulation of Bcl-2/Bax and caspase-3 pathways in the spinal cord following SCI.

Taking these results into account, it was concluded that CAR protected the rat spinal cord from injury. The neuroprotective effect of CAR may be associated with suppressing oxidative stress and inhibiting the eNOS signaling pathway following SCI in rats.

\section{References}

1. Baur JA and Sinclair DA: Therapeutic potential of resveratrol: the in vivo evidence. Nat Rev Drug Discov 5: 493-506, 2006.

2. Takenaga M, Ohta Y, Tokura Y, et al: Lecithinized superoxide dismutase (PC-SOD) improved spinal cord injury-induced motor dysfunction through suppression of oxidative stress and enhancement of neurotrophic factor production. J Control Release 110: 283-289, 2006.

3. Fu J, Fan HB, Guo Z, et al: Salvianolic acid B attenuates spinal cord ischemia-reperfusion-induced neuronal injury and oxidative stress by activating the extracellular signal-regulated kinase pathway in rats. J Surg Res 188: 222-230, 2014.

4. Zhou YF, Li L, Feng F, et al: Osthole attenuates spinal cord ischemia-reperfusion injury through mitochondrial biogenesis-independent inhibition of mitochondrial dysfunction in rats. J Surg Res 185: 805-814, 2013.

5. Diaz-Ruiz A, Vergara P, Perez-Severiano F, et al: Cyclosporin-A inhibits constitutive nitric oxide synthase activity and neuronal and endothelial nitric oxide synthase expressions after spinal cord injury in rats. Neurochem Res 30: 245-251, 2005.

6. Guimarães AG, Xavier MA, de Santana MT, et al: Carvacrol attenuates mechanical hypernociception and inflammatory response. Naunyn Schmiedebergs Arch Pharmacol 385: 253-263, 2012.

7. Guimarães AG, Oliveira GF, Melo MS, et al: Bioassay-guided evaluation of antioxidant and antinociceptive activities of carvacrol. Basic Clin Pharmacol Toxicol 107: 949-957, 2010.

8. Yu W, Liu Q and Zhu S: Carvacrol protects against acute myocardial infarction of rats via anti-oxidative and anti-apoptotic pathways. Biol Pharm Bull 36: 579-584, 2013.

9. Deng W, Lu H and Teng J: Carvacrol attenuates diabetes-associated cognitive deficits in rats. J Mol Neurosci 51: 813-819, 2013.

10. Ravikumar R, Fugaccia I, Scheff SW, Geddes JW, Srinivasan C and Toborek M: Nicotine attenuates morphological deficits in a contusion model of spinal cord injury. J Neurotrauma 22: 240-251, 2005.

11. Basso DM, Beattie MS, Bresnahan JC, et al: MASCIS evaluation of open field locomotor scores: effects of experience and teamwork on reliability. Multicenter Animal Spinal Cord Injury Study. J Neurotrauma 13: 343-359, 1996.

12. Springer JE, Azbill RD and Knapp PE: Activation of the caspase-3 apoptotic cascade in traumatic spinal cord injury. Nat Med 5: 943-946, 1999.

13. Sharma HS, Gordh T, Wiklund L, Mohanty S and Sjöquist PO: Spinal cord injury induced heat shock protein expression is reduced by an antioxidant compound H-290/51. An experimental study using light and electron microscopy in the rat. J Neural Transm 113: 521-536, 2006.

14. Harman D: Free radical theory of aging: an update: increasing the functional life span. Ann NY Acad Sci 1067: 10-21, 2006.

15. Die J, Wang K, Fan L, Jiang Y and Shi Z: Rosuvastatin preconditioning provides neuroprotection against spinal cord ischemia in rats through modulating nitric oxide synthase expressions. Brain Res 1346: 251-261, 2010.

16. Nakajima H, Uchida K, Yayama $\mathrm{T}$, et al: Targeted retrograde gene delivery of brain-derived neurotrophic factor suppresses apoptosis of neurons and oligodendroglia after spinal cord injury in rats. Spine (Phila Pa 1976) 35: 497-504, 2010.

17. Shacka JJ and Roth KA: Regulation of neuronal cell death and neurodegeneration by members of the Bcl- 2 family: therapeutic implications. Curr Drug Targets CNS Neurol Disord 4: 25-39, 2005. 